\title{
MONITORAMENTO DE INSETOS EM ESTRUTURA ARMAZENADORA ATRAVÉS DE ARMADILHA COM ATRATIVO ALIMENTAR LOCALIZADA FORA DOS SILOS
}

\author{
Insects Sampling in Storage Structure Using a Trap With Food \\ Attractant Placed Outside the Silos
}

\author{
Airton Rodrigues Pinto Junior ${ }^{1}$ \\ Fabiane Ceruti ${ }^{2}$ \\ Saulo Henrique Weber ${ }^{3}$
}

\section{Resumo}

O monitoramento das populações de insetos associados com grãos armazenados é fundamental para a detecção precoce, acompanhamento da evolução das infestações e para a tomada de decisão sobre as medidas de controle. $\mathrm{O}$ objetivo geral dessa pesquisa foi avaliar uma técnica para o monitoramento de insetos em estrutura armazenadora de grãos e estabelecer as áreas de risco de infestação. $O$ trabalho foi realizado em 3 silos da Fazenda Gralha Azul - PUCPR com capacidade de 1200 t, no período de novembro de 2003 a maio de 2004. Foram utilizadas 4 armadilhas do tipo gaiola com atrativo alimentar, distribuídas na área externa do silo. A cada quinze dias, as armadilhas foram verificadas para a contagem e identificação dos insetos. Foram capturados, nas quatro armadilhas, durante o período avaliado, 1595 insetos das ordens Coleoptera e Psocoptera. As espécies encontradas em maior número foram: Sitophilus zeamais (Coleoptera: Curculionidae), 995, e Carpophilus sp. (Coleoptera: Nitidulidae), 294 indivíduos capturados. Por meio de análise de variância e teste de Tukey observou-se que as gaiolas 3 e 1 tiveram capturas estatisticamente significantes relativas ao número de insetos. Desta forma, o monitoramento, utilizando armadilha gaiola, representa uma ferramenta indispensável para a detecção prévia das populações de insetos, permitindo o direcionamento da higienização das estruturas e de outras estratégias de manejo para a manutenção da qualidade de grãos armazenados.

Palavras-chave: Controle; Insetos; Armazenamento; Ecologia.

1 Pontifícia Universidade Católica do Paraná - PUCPR, BR 376 km14 Costeira, São José dos Pinhais, PR C. Postal 129, 83010-500, airton.junior@pucpr.br

2 Universidade Federal do Paraná - UFR, Setor de Ciências Biológicas, Departamento de Zoologia, CP 19020, Curitiba, PR, 81531980, biaceruti@ufpr.br

3 Pontifícia Universidade Católica do Paraná - PUCPR, BR 376 km14 Costeira, São José dos Pinhais, PR C. Postal 129, 83010-500, s.h.w@bol.com.br 


\section{Abstract}

The monitoring of stored grain insect populations is basic for the preventive detection, following up the infestation evolution and for control decisions. The general objective of this research was to evaluate a technique for the monitoring of insects and to establish the infestation areas. The tests were carried in 3 silos of Gralha Azul Experimental Farm - PUC/PR with capacity of $1200 \mathrm{t}$, in the period of November 2003 to May 2004. Four cage traps with food attractant, placed outside the silos, were evaluate. Every fifteen days, the traps were verified for the count and identification of the insects. They were captured in the four traps during the period appraised 1595 insects of the orders Coleoptera and Psocoptera. The most abundant species were: Sitophilus zeamais (Coleoptera: Curculionidae), 995, and Carpophilus sp. (Coleoptera: Nitidulidae), 294 collected specimens. Through analysis of variance and Tukey test it was observed that the cages 3 and 1 presented statistical significance with respect to number of insects capture. The monitoring, using trap cage, represents an indispensable tool for the previous detection of the populations of insects, allowing to the conduct the cleaning and other control measures to maintain the grain quality during storage.

Keywords: Control; Insects; Storage; Ecology.

\section{Introdução}

O manejo integrado de pragas (MIP) no armazenamento deve ser baseado no entendimento do ecossistema peculiar, relativamente fechado de um silo ou armazém, considerando a dinâmica, comportamento e biologia das espécies, bem como os fatores que regulam as populações. O monitoramento é um dos fundamentos do MIP e propõe o uso de técnicas que geram informações importantes sobre a presença e flutuação de insetos para a tomada de decisões sobre as medidas de controle mais adequadas (DOWDY; MCGAUGHEY, 1994).

As estratégias de manejo devem incluir manutenção e limpeza das estruturas e um conhecimento mais profundo dos padrões técnicos considerados ideais quanto à segurança e operacionalidade para evitar danos qualitativos e quantitativos, desde o momento da colheita até o armazenamento.

As perdas na armazenagem dos grãos podem variar bastante, dependendo das condições do armazenamento. Considera-se que $80 \%$ do produto armazenado sofrem perda de $0,15-0,30 \%$ ao ano, devido à perda de umidade, consumo por fungos e insetos, aeração deficiente, transporte e outras práticas inadequadas (LAZZARI, 2002). Como exemplo, maquinários e implementos de colheita e beneficiamento desregulados ou mal manejados resultam em quebras técnicas de armazenagem (LAZZARI; LAZZARI, 2001).

A massa de grãos proporciona alimento abundante para os insetos e um ambiente moderadamente protegido para o seu desenvolvimento (SINHA, 1995). De acordo com o hábito alimentar, os insetos pragas de grãos armazenados podem ser classificados como pragas primárias, capazes de atacar os grãos inteiros e sadios, e secundárias, que se alimentam dos grãos previamente danificados mecanicamente ou por insetos primários (PACHECO; PAULA, 1985). Segundo os mesmos autores, são exemplos de pragas primárias, Rhyzopertha dominica (Fabricius, 1792) (Coleoptera: Bostrichidae), Sitophilus oryzae (Linnaeus, 1763) e Sitophilus zeamais Motschulsky, 1855 (Coleoptera: Curculionidae), Sitotroga cerealella (Oliver, 1819) (Lepidoptera: Gelechiidae). As pragas secundárias mais comuns em armazenagem são as espécies Oryza ephilus surinamensis (Linnaeus, 1758) (Coleoptera: Silvanidae), Tribolium castaneum (Herbst, 1797), Cadra cautella (Walker, 1863) e Corcyra cephalonica (Staiton, 1866) (Lepidoptera: Pyralidae). Os psocópteros (Psocoptera) mais comuns no ambiente de armazenamento pertencem à família Liposcelidae representadas pelo gênero Liposcelis.

A detecção ou estimativa de insetos associados com grãos armazenados é fator importante na tomada de decisão de práticas de manejo integrado de pragas (HAGSTRUM; FLINN, 1992). Seja qual for o de método usado para a detecção de insetos, a interpretação dos dados é fundamental para seu uso efetivo. Variáveis ambientais, atrativos alimentares e feromônios afetam diretamente o comportamento dos insetos e conseqüentemente têm influência na eficiência de métodos de monitoramento (WRIGHT; HAGSTRUM, 1990). O desenvolvimento de diferentes tipos de armadilhas para a amostragem de insetos de produtos armazenados, juntamente com o progresso na identificação e síntese de feromônios e atrativos para as principais espécies de insetos, têm sido alvo de muitas pesquisas nas duas últimas décadas (PINNIGER, 1990). 
Segundo Barak et al. (1990), as armadilhas para insetos de produtos armazenados enquadram-se em três categorias: armadilhas para captura de insetos aéreos, incluindo as adesivas e as de funis; armadilhas de superfície, para capturar insetos enquanto andam (com feromônios ou atrativos alimentares) e as armadilhas usadas na massa de grão, como as do tipo calador (PEREIRA, 1994). Essas categorias se tornam menos distintas à medida que as armadilhas são usadas para espécies diferentes daquelas para as quais foram desenvolvidas.

As armadilhas tipo gaiola com atrativos alimentares não são armadilhas no senso estrito, uma vez que atraem os insetos, mas não evitam sua fuga voluntária, entretanto, o contato com 0 alimento encoraja os insetos a permanecerem no seu interior (BARAK et al., 1990; PINNIGER, 1990). Estes dispositivos têm sido utilizados com sucesso no monitoramento da atividade de insetos no exterior de silos (THRONE; CLINE, 1994).

A resposta dos insetos às iscas alimentares depende da interação entre o odor dos alimentos e os feromônios. A produção de certos componentes dos feromônios por algumas espécies de insetos pode ser afetada pela presença de certos químicos presentes na dieta e a resposta de alguns insetos aos seus feromônios pode ser melhorada ou sinergizada pela recepção simultânea de odores alimentares (PINNIGER, 1990). As vantagens da utilização de armadilhas com atrativos alimentares são: ambos os sexos respondem ao estímulo, são multiespecíficas, podem atrair adultos e larvas, podem melhorar a resposta aos feromônios e são relativamente simples e baratas. A desvantagem é que a presença de outras fontes de alimento pode afetar a sua eficiência.

O tipo de armadilha a ser adotado depende do objetivo que se quer alcançar. As três principais razões para o uso de armadilhas são: a detecção prematura de pragas para detectar insetos antes que eles sejam encontrados por inspeção visual ou antes que exista evidência de dano; 0 monitoramento do número de insetos para determinar os níveis de captura que possibilitem ações de controle e o levantamento de infestações tanto quantitativa quanto qualitativamente, coletando insetos para identificação, testes de resistência ou avaliando práticas de controle (PINNIGER, 1990). Desta maneira, a técnica de monitoramento pode gerar informações sobre a presença de pragas na área e como indicativo para proceder à amostragem direta de insetos na massa de grãos (DOWDY; MCGAUGHEY, 1994).

Pereira (1999); Paula (2001); Ceruti (2003) avaliaram a armadilha tipo gaiola com atrativo alimentar para o monitoramento preventivo de insetos de produtos armazenados ao redor de unidades armazenadoras. Os resultados mostraram a capacidade deste tipo de armadilha em detectar populações residuais na estrutura armazenadora, auxiliando na determinação dos principais locais com potencialidade para o desenvolvimento de insetos.

O objetivo geral dessa pesquisa foi avaliar uma técnica para o monitoramento de insetos que possa ser utilizada em programas de manejo integrado de pragas de produtos armazenados. Os objetivos específicos foram: avaliar a ocorrência e flutuação de insetos na estrutura de armazenagem, avaliar a eficácia da armadilha tipo gaiola para 0 monitoramento dos insetos, baseando-se no número e espécie de insetos capturados e determinar as áreas de risco de infestação de insetos de acordo com as capturas.

\section{Material e métodos}

\section{Local de realização dos experimentos}

O monitoramento dos insetos foi realizado em 3 silos, com capacidade de 490 toneladas cada, localizados na Fazenda Experimental Gralha Azul da Pontifícia Universidade Católica do Paraná em Fazenda Rio Grande - PR. As coletas foram realizadas quinzenalmente no período de novembro de 2003 a maio de 2004.

\section{Armadilha}

A armadilha utilizada foi a tipo gaiola, para monitoramento de insetos na estrutura de armazenamento, com atrativo alimentar. Foram utilizadas quatro armadilhas construídas com compensado (22 $\mathrm{cm}$ de largura X $30 \mathrm{~cm}$ de comprimento X 15 $\mathrm{cm}$ de altura), cobertas com chapa de ferro galvanizado, em forma de telhado, com a finalidade de proteger 0 atrativo alimentar da exposição direta ao tempo. Nas laterais da estrutura de compensado, foi colocada uma malha metálica para permitir 
a livre entrada dos insetos, mas evitar o ataque de outros organismos, como pássaros e roedores (THRONE; CLNE, 1991). As armadilhas foram distribuídas ao redor da unidade armazenadora.

Nessas armadilhas foram colocados $250 \mathrm{~g}$ de isca alimentar, composta por uma mistura de grãos de milho, trigo, quirera de milho e germe de trigo na proporção 1:1:1:1/2, respectivamente. A isca foi armazenada em freezer até o momento de uso e trocada quinzenalmente nas armadilhas. Os insetos capturados foram retirados, juntamente com a isca, e colocados em placas de Petry para triagem, identificação e quantificação das espécies.

Os dados foram analisados estatisticamente por meio da análise de variância e testes de médias de Tukey a $5 \%$ de probabilidade e utilizando o programa Jump 4.0.

\section{Resultados e discussão}

Foram capturados na estrutura armazenadora, no período de novembro de 2003 a maio de 2004, com as armadilhas tipo gaiola, um total de 1595 insetos, pertencentes às ordens Coleoptera e Psocoptera (TABELA 1).

Os coleópteros de produtos armazenados encontrados em maior número nas armadilhas foram: Sitophilus zeamais (Coleoptera: Curculionidae) com 955, Carpophilus sp. (Coleoptera: Nitidulidae) com 294, Cryptolestes ferrugineus (Coleoptera: Cucujidae) com 115 espécimes, Gnatocerus cornutus (Coleoptera: Tenebrionidae) com 39, Oryzaephilus surinamensis (Coleoptera: Silvanidae) com 31 e Tribolium castaneum (Coleoptera: Tenebrionidae) com 28 espécimes capturados. Liposcelis sp. (Psocoptera: Liposcelidae) teve 133 indivíduos coletados nas armadilhas gaiolas.

\section{TABELA 1 - Número de insetos capturados em quatro armadilhas tipo gaiola com atrativo alimentar, em estrutura armazenadora de grãos, no período de novembro/2003 a maio/2004, na Fazenda Gralha Azul, PUCPR.}

Table 1 - Number of captured insects in four traps type attractive river steamer with alimentary, in storing structure of grains, from november/ 2003 to may/2004, in the Fazenda Experimental Gralha Azul, PUCPR.

\begin{tabular}{lrrr} 
Família & Espécie & Número de insetos & $\%$ \\
\hline Curculionidae & Sitophilus zeamais Motschulsky & 955 & 59,8 \\
Nitidulidae & Carpophilus sp. & 294 & 18,4 \\
Cucujidae & Cryptolestes ferrugineus (Stephens) & 115 & 7,2 \\
Tenebrionidae & Gnatocerus cornutus (F.) & 39 & 2,4 \\
Silvanidae & Oryzaephilus surinamensis (L.) & 31 & 1,9 \\
Tenebrionidae & Tribolium castaneum (Herbst) & 28 & 1,7 \\
\hline Total & & $\mathbf{1 4 6 2}$ & $\mathbf{9 1 , 6}$ \\
\hline
\end{tabular}

O número total de insetos capturados em cada armadilha tipo gaiola durante o período de coleta está demonstrado na Figura 1. Pode-se observar que o maior número de capturas se deu nas armadilhas 3 e 1, com média de captura de 80,57 e 67,57 insetos respectivamente. Pela análise de variância e teste de Tukey (TABELA 2) foi observado que há diferença significativa entre o número de insetos capturados nas armadilhas $(\mathrm{F}=4,49$ e $\mathrm{p}=0,0123)$ de acordo com sua posição na estrutura, indicando a possibilidade de haver infestação no milho armazenado ou focos de infestação na estrutura das células. 
FIGURA 2 - Número total de insetos capturados com 4 armadilhas tipo gaiola com atrativo alimentar, em estrutura armazenadora de grãos na Fazenda Gralha Azul, PUCPR, no período de novembro de 2003 a maio de 2004.

FIGURE 2 - Total number of captured insects with 4 traps type attractive river steamer with alimentary, in storing structure of grains in Fazenda Experimental Gralha Azul, PUCPR, from November/2003 to May/2004.

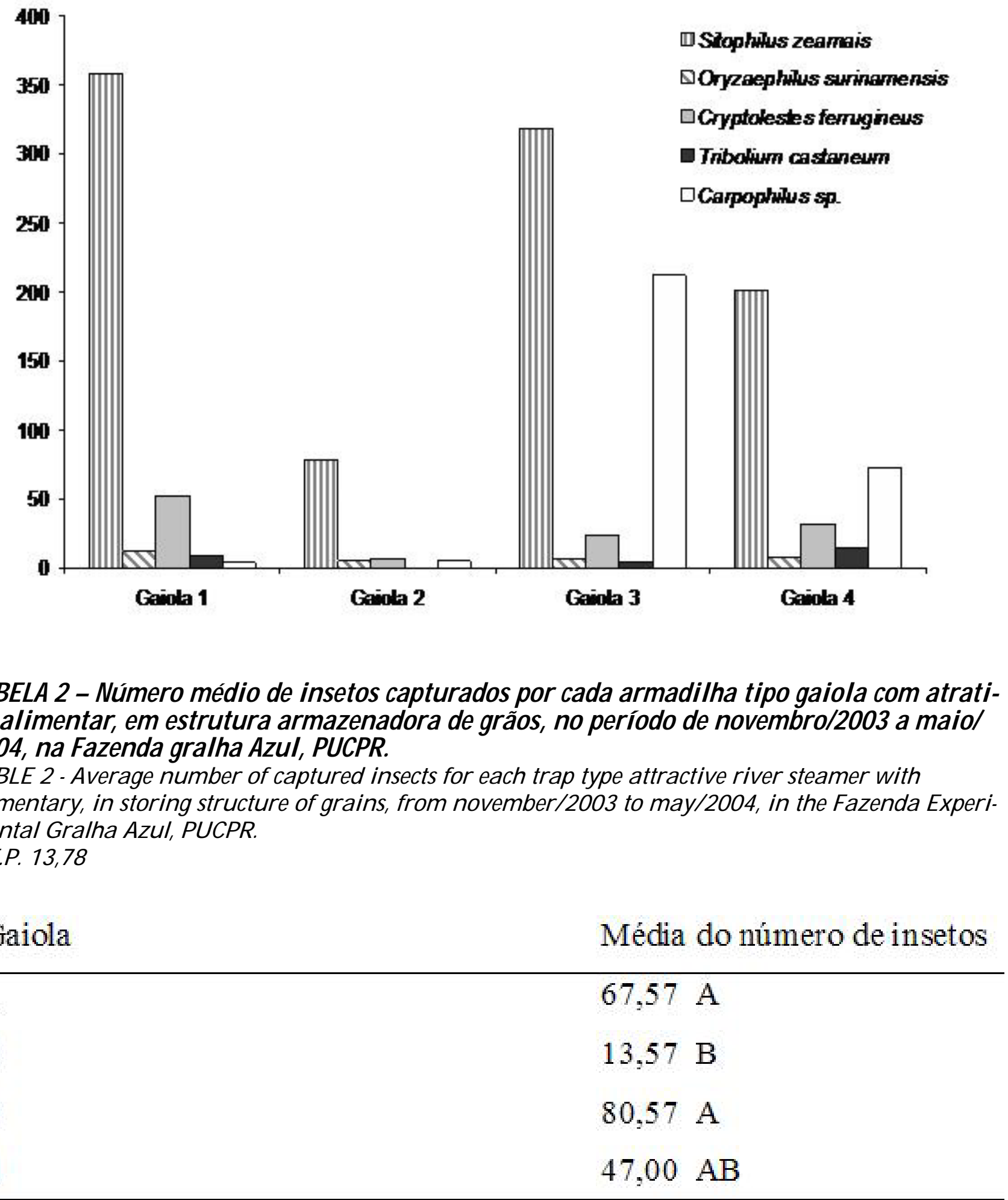


A ocorrência de S. zeamais em grande número (59,8\%) na unidade armazenadora está de acordo com as observações de Pereira et al. (2000), que capturaram 11 espécies de insetos em armadilhas tipo gaiola colocadas ao redor de silos com milho, sendo que S. zeamais representou $63,4 \%$ do total. Esses gorgulhos podem iniciar a infestação na lavoura, principalmente quando ocorre 0 atraso na colheita, ou serem provenientes de lotes infestados vindos de outras unidades armazenadoras.

Muitas espécies de insetos capturadas nas armadilhas com atrativos alimentares produzem feromônios de agregação os quais provavelmente atraem outros indivíduos (COGBURN et al., 1984). Assim, o grande número de insetos capturados pelas armadilhas com atrativo alimentar pode também ser o resultado da atração de outros membros da espécie pela ação de feromônios de agregação. Além disso, membros da família Cucujidae são conhecidos por serem atraídos por odores fúngicos, os quais estão presentes em grande quantidade em grãos e sementes moídos ou triturados (PIERCE et al., 1991; LAZZARI, 1997).

Além de detectar focos de infestação de insetos ao redor de unidades armazenadoras, os dados obtidos com o uso de armadilhas tipo gaiola com atrativo alimentar indicam quando e onde práticas de manejo, como limpeza de estruturas, técnicas preventivas de controle e monitoramento da massa de grãos, devem ser realizadas com maior ênfase. Outro aspecto importante é que, à medida que o registro de dados vai aumentando, é possível estabelecer um histórico da presença de insetos na estrutura, que é muito valioso para fins de manejo integrado de pragas.

Uma vez determinadas as áreas potenciais, buscam-se as prováveis causas da infestação, fazendo-se uma análise detalhada do local, observando desde os aspectos de higienização da estrutura, presença de resíduos de grãos, madeira em decomposição e sementes de plantas não cultivadas. Segundo Linsley (1944), cantos ou superfícies de difícil acesso com resíduos proporcionam o desenvolvimento de populações de insetos (ARBOGAST; MULLEN, 1988).

Segundo Sartori; Lorini (2000), como é sabido há muito tempo, a limpeza de equipamentos e da estrutura de armazenagem de grãos, e também dos veículos utilizados para o transporte, é muito importante para reduzir a infestação por insetos, no entanto, essa ainda não tem sido uma prática corriqueira.

Os resultados obtidos durante esse período de 6 meses de coleta forneceram informações importantes para a implantação de um programa de manejo integrado de pragas na unidade em questão. Com esses dados, pode-se determinar os locais que necessitavam de maior atenção quanto à sanidade e também planejar estratégias para prevenir futuras infestações.

\section{Conclusões}

As quatro armadilhas gaiolas utilizadas para o monitoramento de insetos no silo capturaram as principais pragas primárias e secundárias que ocorrem em produtos armazenados como: Sitophilus zeamais, Cryptolestes ferrugineus, Oryzaephilus surinamensis e Tribolium castaneum.

As armadilhas com atrativo alimentar capturaram um número elevado de insetos, sendo, portanto, instrumentos valiosos para detectar populações residuais de insetos.

As capturas com as armadilhas indicaram uma infestação extremamente alta de S. zeamais, indicando a necessidade urgente de higienização da estrutura e controle direcionado a esta espécie.

Os dados obtidos a partir das coletas com as armadilhas proporcionam informações que são úteis para a elaboração de mapas de risco, os quais são úteis para programas de manejo integrado de pragas, permitindo direcionar medidas preventivas, como a limpeza da estrutura e maquinário e até medidas de controle das populaçõ es residuais, com a redução considerável dos produtos químicos normalmente aplicados em grãos armazenados.

\section{Referências}

ARBOGAST, R. T; MULEN, M. A. Insect succession in a stored-corn ecosystem in southeast Georgia. Ann. Entomol. Soc. Am. v. 81, p. 899-912, 1988.

BARAK, A. V.; BURKHOLDER, W. E.; FAUSTINI, D. L. Factors affecting the design of traps for stored product insects. J. Kans. Entomol. Soc. v. 63, p. 466-487, 1990. 
CERUTI, F. C. Técnicas de monitoramento e de controle de insetos em milho armazenado. Curitiba, 2003, 93 f. Dissertação (Mestrado em Zoologia) - Universidade Federal do Paraná, Curitiba, 2003.

COGBURN, R. R.; BURKHOLDER, W.E.; WILLIAMS, $\mathrm{H}$. J. Field tests with the aggregation pheromone of the lesser grain borer (Coleoptera: Bostrichidae). Environ. Entomol. v. 13, p. 162-166, 1984.

DOWDY, A. K.; MCGAUGHEY, W. H. Seasonal activity of stored product insects in and around farm-stored wheat. J. Econ. Entomol. v. 87, p. 1352-1358, 1994.

HAGSTRUM, D. W.; FLINN, P. W. Integrated pest managementof stored insects. In:__ . Storage of cereal grains and their products. D.B. Sauer (ed.) Am. Ass. Cereal Chem. MN, EUA, p. 535-562. 1992.

LAZZARI, F. A. Umidade, fungos e micotoxinas na qualidade de sementes, grãos e rações. Edição do autor. Curitiba, 1997.

LAZZARI, F. A.; LAZZARI, S. M. N. Identificação, quantificação e interpretação de perdas em produtos armazenados. Revista Granos \& Postcosecha Latino Americana, v. 6, n. 25, 2001.

LINSLEY, E. G. Natural sources, habitats, and reservoirs of insects associated with stored food products. Hilgardia: A Journal Of Agricultural Science, v.16, n. 3, p. 187-224, 1944.

PACHECO, I. A.; PAULA, D. C. Insetos de grãos armazenados: identificação e biologia. Campinas: Fundação Cargill, 1995.

PAULA, M. C. Z. Manutenção da qualidade do arroz armazenado: monitoramento e controle de insetos. Curitiba, 2001. 74 f. Tese (Doutorado em Entomologia) - Universidade Federal do Paraná, UFPR, 2001.

PEREIRA, P. R. V. S. Comparação entre dois métodos para a detecção de coleópteros adultos (Insecta: Coleoptera) e ocorrência de fungos em trigo armazenado. Curitiba, PR. 1994. 62 f. Dissertação (Mestrado em Zoologia) - Universidade Federal do Paraná, 1994.
PEREIRA, P. R. V. S. Contribuição para o manejo integrado de pragas de produtos armazenados. Curitiba, PR, 1999. 99 f. Tese (Doutorado em Entomologia) - Universidade Federal do Paraná, UFPR, 1999.

PEREIRA, P. R. V. S.; LAZZARI, F.; LAZZARI, S. M. $\mathrm{N}$. Insect monitoring outside grain storage facilities in southern Brazil. In: INTERNATIONAL WORKING CONFERENCEONSTORED-PRODUCT PROTECTION, 7., 2000. Proceedings... 2000. v. 2. p. 1534-1536.

PIERCE, A. M., et al. C. Fungal volatiles: semiochemicals for stored-product beetles (Coleoptera: Cucujidae). J. Chem. Ecol. v. 17, p. 581-597, 1991.

PINNIGER, D. B. Food- baited traps: Past, present and future. J. Kans. Entomol. Soc. v. 63, p. 533538, 1990.

SARTORI, M. R.; LORINI, I. Pesticide resistence in stored grain pests in Brazil: Strategies for management. In: __ INTERNATIONAL WORKING CONFERENCEONSTORED-PRODUCT PROTECTION, 7., 2000. Proceedings... 2000. v. 1. p. 966-973.

SINHA, R. N. The stored-grain ecosustem. New York: Marcel Dekker, 1995.

THRONE, J. E.; CLINE, L D. Seasonal abundance of maize and rice weevils (Coleoptera: Curculionidae) in South Carolina. J. Agric. Entomol. v. 8, p. 93100, 1991.

THRONE, J. E.; CLINE, L. D. Seasonal flight activity and seasonal abundance of selected stored-product coleoptera around grain storages in South America. J. Agric. Entomol. v. 11, p. 321-338, 1994.

WRIGHT, V. F.; HAGSTRUM, D. W. Trapping technology formonitoring stored-products insects. J. Kans. Entomol. Soc. v. 63, p. 464-465, 1990. 